\title{
Influence of anion-water interactions on the behaviour of lipases in room temperature ionic liquids
}

\begin{abstract}
In this report, molecular dynamics simulations were applied in order to investigate the effect of Room Temperature Ionic Liquid (RTIL) anions toward the structure and dynamic properties of lipases. Two lipases were studied; Candida antarctica lipase B and Candida rugosa lipase were solvated by five RTILs that contained the same cation, with increasing hydration levels. Several properties were investigated: structural deviations and flexibility of the protein conformation, the behaviour of RTILs at the protein surface, and the interactions between RTILs and water molecules in the systems. Both lipases' conformations showed an increased structural stability in RTILs when compared to an aqueous solution. The lowest structural deviation was observed around 15 to 20 percent of water content (w/w protein). The RTIL with the chloride anion was shown to be the exception however, inducing the least structural stability at low water percentages. The flexibility of both lipases was clearly affected when transferred from aqueous into RTILs. The flexible regions found for both lipases in water were significantly more rigid in RTILs. Around the protein surface, the behaviour of RTIL anions and the water molecules was similar to other conventional organic solvents. The water retention ability for all RTIL anions was consistent for both lipases except for the bis(trifluoromethylsulfonyl)imide anion, which showed distinctive behaviour toward different protein surface properties. The effect of water content was more profound compared to the difference between the RTILs anions studied. However, it was found that the structural and dynamic properties of the lipases were affected by the behaviour of anions toward the hydration layer of the enzymes.
\end{abstract}

Keyword: Room temperature ionic liquids (RTIL); Lipases; Anion; Water 